$\Phi=$ 核

\title{
The 1000 lumbar spine radiograph study
}

\author{
Glorianne Pullicino ${ }^{1,2}$ *, Jessica Pavia ${ }^{1}$, Paul Sciortino ${ }^{1}$, Ahmed Chilmeran ${ }^{3}$, \\ Philip Sciortino ${ }^{1}$, Richard Pullicino ${ }^{4}$ \\ ${ }^{1}$ Department of Family Medicine, University of Malta, Malta \\ ${ }^{2}$ Department of Primary Health Care, Floriana, Malta \\ ${ }^{3}$ Mater Dei Hospital, Msida, Malta \\ ${ }^{4}$ The Walton Centre, Liverpool, United Kingdom \\ *Corresponding author E-mail: glorianneb@gmail.com
}

\begin{abstract}
Magnetic resonance imaging is being increasingly used to optimize the diagnostic process for low back pain and to manage the risk of missing life-threatening pathology. The aim of the study was to examine the care pathway of low back pain with respect to the utilisation of CT and MRI service utilisation. A descriptive, retrospective, cross-sectional study was performed. A random sample of 1000 primary care patients presenting with low back pain who underwent lumbar spine radiography within a specified period was explored. $20 \%$ $(n=198)$ of patients who underwent lumbosacral spine X-ray were referred for MRI investigation. Subsequently, 15 (7.6\%) patients underwent joint infiltration whilst $6(3 \%)$ patients underwent neurosurgical intervention during 2 years of follow-up. Such findings provide information for policy makers about the utility of MRI and CT scans.
\end{abstract}

Keywords: Community Health Systems; Health Services Research; Low Back Pain; Primary Care; Radiology.

\section{Introduction}

The causes of low back pain can be challenging to differentiate because of the similarities in presentation (Arnbak et al. 2015). Even though low back pain is one of the most prevalent disorders in primary care, very often, a definite diagnosis cannot be formulated early enough to either reassure the patient or to refer appropriately for non-conservative treatment options (Arnbak et al. 2015, Keshtkaran et al. 2012).

Moreover, there is a little consensus among the specialists about appropriate low back pain procedures (Keshtkaran et al. 2012). Therefore, more knowledge is required about the various causes of low back pain to improve its management (Arnbak et al. 2016). Magnetic resonance imaging (MRI) is being increasingly used in an attempt to optimize the diagnostic process for low back pain (Arnbak et al. 2015, 2016, Keshtkaran et al. 2012). The ultimate benefit of an MRI depends upon the choice of treatment option given the current clinical condition and increasingly the expectations of the patient.

In Malta, direct access to MRI is not available to public primary care physicians. Primary care is provided by the state health service in governmental Health centres and by private primary care physicians in community pharmacies or within their own offices. The public service is free at the point of use (Pullicino et al. 2015). Primary care doctors have a gate-keeper role (Schäfer 2016). All doctors can refer patients to perform plain radiography in public Health centres. Various uncertainties remain regarding the use of MRI or CT imaging after performing lumbosacral spine radiography in primary care.

Therefore, further research is essential to expand the knowledge of radiological findings visualized by MRI or CT post-lumbosacral spine plain radiography performed in a primary care setting and the subsequent course of low back pain that necessitates non- conservative management. There are still little evidence-based findings that support the recommendation for further radiological investigations post-lumbar spine radiography in primary care patients.

The aim of the study was to examine CT and MRI service utilization and findings after a lumbosacral spine radiograph request by the primary care physician and to examine subsequent health care services utilization.

\section{Methods}

A descriptive, retrospective, cross-sectional study design was applied. All requests for lumbosacral spine radiographs taken in a public primary healthcare center between January and December 2014 were obtained from the Radiology Information System (RIS) and the Picture Archiving and Communication System (PACS). The data was acquired in an anonymous manner.

A random sample of 1000 primary care patients presenting with low back pain who underwent lumbar spine radiography was selected. The patients' demographic and clinical characteristics and any subsequent radiological investigations were compiled and categorized. A focus group composed of primary care clinicians, and a radiologist $(n=4)$ was conducted to analyses subsequent MRI findings and categorize them. Emergent themes and categories were identified. Data analysis was carried out using the Statistical Package for Social Sciences Version 20.

Patients who underwent lumbosacral spine radiography in the private sector and in the public hospitals were excluded from this study. Ethical approval was obtained from the University of Malta Research Ethics committee. 


\section{Results}

The majority of the participants undergoing lumbosacral spine radiography in primary care were females $(54 \%, \mathrm{n}=536)$. The sample population had an age distribution of 8-96 years with a mean of 54.1 years \pm 17.2 years.

At the time of the study, 20\% $(n=198)$ of primary care patients who underwent lumbosacral spine X-ray, subsequently had an MRI investigation from the secondary care sector.55\% $(n=109)$ of these patients were females. This population had an age distribution of 13-96 years with a mean of 53.5 years \pm 15.3 years.

Table 1 shows the frequencies of MRI patterns in the findings. The commonest finding was that of disc pathology $(78.7 \%$, $\mathrm{n}=156$ ), 37 of which had narrowing of spinal canal and 18 had spondylolisthesis. Facet joint pathology occurred in 85 instances whilst degenerative spinal disease was found in 52 cases. Bone lesions included a case of diffuse infiltrations of the vertebral bodies throughout the whole spine and 1 case of multiple bone marrow metastases. Benign neoplasm included 1 case S1 nerve root schwannoma. 250 lumbar spine radiographs were required to find 1 fracture on subsequent MRI. Out of the 4 detected fractures, there was 1 old collapse fracture of the upper endplate of L1. One patient refused the investigation due to claustrophobia.

\begin{tabular}{lll}
\multicolumn{3}{c}{ Table 1: The Frequencies of Different MRI Findings } \\
\hline MRI Finding & Number of cases & $\%$ \\
\hline Disc Pathology & 156 & 78.7 \\
Fracture & 4 & 2 \\
Benign Neoplasm & 1 & 0.5 \\
Bone lesion & 2 & 1 \\
Spinal cord pathology & 1 & 0.5 \\
Other & 34 & 17.2 \\
\hline
\end{tabular}

Following lumbar spine radiography, 7 primary care patients underwent CT Spine. There were 3 cases of L1 fracture and 1 case of T12 fracture. $1 \mathrm{CT}$ showed limbus vertebra of L4 whilst another CT showed grade I spondylolisthesis at the L5/S1 level with neural arch defects at the sacral vertebrae except S1. No abnormalities were reported in 1 case.

There was a statistically significant difference between age and spinal canal pathology $\chi^{2}(82, \mathrm{n}=1000)=117.7, \mathrm{p}=0.006$, phi $=$ 0.343 . Such pathology was most prevalent in the 60-70-year age group. On the other hand, there were no significant differences between age and spinal cord pathology, disc pathology, facet joint arthropathy, fractures, neoplasms and spondylolisthesis.

A statistically significant difference was noted between degenerative spinal disease and age $\chi^{2}(8, \mathrm{n}=198)=21.78, \mathrm{p}=0.005$, phi $=0.331$. This pathology was most frequent inpatients aged over 60 years. Degenerative spinal disease tended to occur more common in males but there was no statistically significant difference.

Furthermore, a statistically significant difference was noted between gender and fractures found on MRI post-lumbosacral spine $\mathrm{X}-\operatorname{ray} \chi^{2}(1, \mathrm{n}=1000)=4.64, \mathrm{p}=0.031$, phi $=-.068$. Fractures found on MRI investigation occurred in male primary care patients. These patients were all in the 65-80-year age group. However, there were no significant differences between gender and neoplasms, spondylolisthesis, disc pathology, facet joint arthropathy, spinal cord and canal pathology.

Furthermore, of the 198 patients who had a lumbosacral spine MRI scan ordered from the secondary care sector, 15 (7.6\%) patients underwent lumbosacral joint infiltration whilst $6(3 \%)$ patients underwent neurosurgical intervention during 2 years of follow-up.

\section{Discussion}

The participants were predominantly females. This might be because females have higher GP service utilization rate (West rate (West et al. 2010, Pullicino et al. 2015, 2016). The mean age and range of the patient population undergoing MRI were similar to those reported in another study (53.5 years vs. 50.45 years, 13-96 years vs. 8-87 years) (West et al. 2010). Both studies showed that disc pathology was a highly prevalent MRI finding (West et al. 2010).

Similar to the current study, a retrospective analysis showed that degenerative disc disease occurred more frequently in older patients with no significant association with gender (West et al. 2010). On the other hand, the other hand, a recent Danish study showed that all degenerative spinal findings were most frequent in men. This was explained by heavy work being more frequent among Danish men compared with women (Arnbak et al. 2015).

This current study demonstrated that spinal canal pathology was mostly prevalent in the 60-70-year age group. Similarly, a systematic review and meta-analysis showed that MR imaging evidence of disc bulge, degeneration, extrusion, protrusion and spondylolysis are more likely to occur in adults 50 years of age or younger with back pain when compared with asymptomatic individuals (Brinjikji et al. 2015).

Fractures found on MRI post-lumbosacral spine X-ray occurred in men over 65 years of age. This might be because osteoporosis is generally thought of as a "woman's disease." The prevalence of osteoporosis and the rate of fractures are much higher in postmenopausal women than in older man (Cawthon 2011). Moreover, men tend to have worse outcomes after fracture(Cawthon 2011). Thus, this could lead to investigate further by requesting an MRI after a lumbosacral spine X-ray. This might give rise to gender and health inequalities due to a diagnostic gender-based bias. Therefore, multidisciplinary interventions for screening and treatment of osteoporosis need to be developed and improved.

A Canadian retrospective, cohort study analysed health care services use after MRI scan of the lumbosacral spine ordered by a primary-care physician. $6.5 \%$ of those patients who underwent MRI scan received spine surgery during 3 years of follow-up. In current study, for every 100 patients who had a lumbosacral spine MRI scan ordered from the secondary care sector, 8 patients underwent lumbosacral joint infiltration whilst 3 patients underwent neurosurgical intervention during 2 years of follow-up. Both studies revealed that most patients receiving MRI scans of the spine do not receive subsequent surgery. This shows that MRI findings on their own would not have significantly changed the management of the patient unless one adds the clinical findings. The significant change beyond a conservative approach despite the use of these imaging modalities still depend largely on the clinical findings and the progress over a defined period.

This hints at the idea that plain lumbosacral spine radiography and MRI findings may not be the most important factors that will determine progress to surgery but also patients' expectations, pain levels, thresholds to pain and clinicians dealing with uncertainty. This leads to the next few questions. When does a lumbosacral spine X-ray or MRI become useful in clinical management and therefore, what should GPs discuss with their patients once they raise the X-ray/MRI question? Further research can delve into this. A modified request form for lumbosacral spine plain radiology should help in the acqusition of high quality structured clinical data which together with the radiological findings and subsequent cause of the low back pain, will help to identify those patients who would need more intensive imaging or faster access to secondary care. This would allow better use of secondary resources, including MRI and CT scan imaging with a therapeutic option beyond conservative management. History of trauma, previous low back pain episodes, duration of current episodes and functional limitations could be included in the request form.

Due to time and resource constraints, this study did not capture the radiological investigations performed in the private sector and in the sole public hospital in Malta. Moreover, patients who had undergone lumbosacral spine radiography in the Emergency Department might have been self-referred from the community. Some lumbosacral spine plain radiographs might have been vetted by the radiographer on-call. Furthermore, this study did not consider cost-effectiveness or patient satisfaction and expectations regarding the value of further imaging after lumbosacral spine 
radiography. It is important to study this aspect to avoid inappropriate and unnecessary imaging. Further research can address these limitations.

Health system analyses are usually cross-sectional. Therefore, it is challenging to come to cause and effect interferences. Pooled cross-sectional and time series offer a possible solution. However, this pre-supposes a more complex system of monitoring policies and their outcomes (Groenewegen 2013).

\section{Conclusions}

The MRI findings on their own would not have significantly changed the management to the patient unless one adds the clinical scenario. These findings would be useful to inform primary care physicians, policy makers and health service researchers aim to support the primary health care system.

\section{Acknowledgements}

Ethical approval was obtained from the University of Malta Research Ethics committee.

\section{References}

[1] Arnbak B, Jensen RK, Manniche C, Hendricks O, Kent P, Jurik AG and Jensen TS (2016) Identification of subgroups of inflammatory and degenerative MRI findings in the spine and sacroiliac joints: a latent class analysis of 1037 patients with persistent low back pain. Arthritis Research \& Therapy, 18(1), p.2163. https://doi.org/10.1186/s13075-016-1131-x.

[2] Arnbak B, Jensen TS, Egund N, Zejden A, Hørslev-Petersen K Manniche C and Jurik AG (2015) Prevalence of degenerative and spondyloarthritis-related magnetic resonance imaging findings in the spine and sacroiliac joints in patients with persistent low back pain. European Radiology, 26(4), pp.1191-1203. https://doi.org/10.1007/s00330-015-3903-0.

[3] Brinjikji W, Diehn FE, Jarvik JG, Carr CM, Kallmes DF, Murad MH and Luetmer PH (2015) MRI Findings of Disc Degeneration are More Prevalent in Adults with Low Back Pain than in Asymptomatic Controls: A Systematic Review and Meta-Analysis. AJNR. American journal of neuroradiology, 36(12), pp.2394-2399. https://doi.org/10.3174/ajnr.A4498.

[4] Cawthon PM (2011) Gender Differences in Osteoporosis and Fractures. Clinical Orthopaedics and Related Research®, 469(7), pp.1900-1905. https://doi.org/10.1007/s11999-011-1780-7.

[5] Groenewegen PP (2013) Analyzing European health systems: Europe as a research laboratory. European journal of public health. https://doi.org/10.1093/eurpub/ckt032.

[6] Keshtkaran A, Bagheri MH, Ostovar R, Salari H, Farokhi MR, Esfandiari A and Yousefimanesh H (2012) Developing Criteria for Lumbar Spine Magnetic Resonance Imaging (MRI) Using RAND Appropriateness Method (RAM). Iranian journal of radiology: a quarterly journal published by the Iranian Radiological Society, 9 (3), pp.130-138. https://doi.org/10.5812/iranjradiol.4063.

[7] Pullicino G, Sciortino P, Calleja N, Schäfer W, Boerma W and Groenewegen $\mathrm{P}(2015)$ Comparison of patients' experiences in public and private primary care clinics in Malta. European journal of public health, 25 (3), pp.399-401. https://doi.org/10.1093/eurpub/cku188.

[8] Pullicino G, Sciortino P, Camilleri L, Schäfer W and Boerma W(2016) The Influence of Patient Characteristics on HealthcareSeeking Behavior: A Multilevel Analysis of 70 Primary Care Practices in Urban-Suburban Regions in Malta.Quality in primary care, 24 (3), pp.106-110.

[9] Schäfer WLA (2016) Primary care in 34 countries: perspectives of general practitioners and their patients. NIVEL.

[10] West W, West KP, Younger EN and Cornwall D (2010) Degenerative disc disease of the lumbar spine on MRI. West Indian Medical Journal, 59(2), pp.192-195. 